CHENYU ZHOU, Ph.D. ${ }^{1}$

E-mail: feibode@hotmail.com

XUAN ZHAO, Ph.D. ${ }^{1}$

(Corresponding author)

E-mail: zhaoxuan@chd.edu.cn

QIANG YU, Ph.D. ${ }^{1}$

E-mail: qiangyu@chd.edu.cn

RONG HUANG, M.S. ${ }^{1}$

E-mail: huangrong@chd.edu.cn

${ }^{1}$ Chang'an University

South Campus of Chang'an University

Chang'an Middle Road, Xi'an, China
Traffic Management Original Scientific Paper Submitted: 5 Mar. 2020 Accepted: 7 Oct. 2020

\title{
DYNAMIC BAYESIAN NETWORK-BASED ESCAPE PROBABILITY ESTIMATION FOR COACH FIRE ACCIDENTS
}

\section{ABSTRACT}

Coach emergency escape research is an effective measure to reduce casualties under serious vehicle fire accidents. A novel experiment method employing a wireless transducer was implemented and the head rotation speed, rotation moment and rotation duration were collected as the input variables for the classification and regression tree (CART) model. Based on this model, the classification result explicitly pointed out that the exit searching efficiency was evolving. By ignoring the last three unimportant factors from the Analytic Hierarchy Process (AHP), the ultimate Dynamic Bayesian Network (DBN) was built with the temporal part of the CART output and the time-independent part of the vehicle characteristics. Simulation showed that the most efficient exit searching period is the middle escape stage, which is 10 seconds after the emergency signal is triggered, and the escape probability clearly increases with the efficient exit searching. Furthermore, receiving emergency escape training contributes to a significant escape probability improvement of more than 10\%. Compared with different failure modes, the emergency hammer layout and door reliability have a more significant influence on the escape

Table 1 - Serious fire accident cases from 2010 in China probability improvement than aisle condition. Based on the simulation results, the escape probability will significantly drop below 0.55 if the emergency hammers, door, and aisle are all in a failure state.

\section{KEYWORDS}

coach fire escape safety; dynamic Bayesian network; classification and regression tree; escape behavior experiment; escape probability estimation.

\section{INTRODUCTION}

Vehicle fire hazards on coaches resulting from collision or self-ignition account for massive losses of lives and economic damage in China [1]. Most of the traffic accidents involving buses and coaches accompanied by fire on the highway that happened from 2010 are collected in Table 1. These data are consistent with the notion that due to a large number of passengers, the casualties are much higher and are more likely to be affected by comprehensive

\begin{tabular}{||c|c|c|c|c||}
\hline Description & Casualty & Time & Location & Road type \\
\hline \hline Self-ignition & 2 fatal; 14 injuries & 21 Jul. 2010 16:46 & Hunan & highway \\
\hline Self-ignition & 26 fatal; 28 injuries & 22 Mar. 2019 19:15 & Hunan & highway \\
\hline Crash + fire & 35 fatal; 13 injuries & 26 Jun. 2016 10:20 & Hunan & highway \\
\hline Self-ignition & 41 fatal; 6 injuries & 22 Jul. 2011 04:00 & Henan & highway \\
\hline Self-ignition & 10 fatal; 17 injuries & 05 Mar. 2014 07:05 & Jilin & highway \\
\hline Crash + fire & 12 fatal; 6 injuries & 16 Jan. 2014 15:52 & Shandong & highway \\
\hline Crash + fire & 22 fatal; 13 injuries & 25 Sep. 2015 03:55 & Hunan & highway \\
\hline Crash + fire & 6 fatal; 31 injuries & 30 Jun. 2019 16:40 & Inner Mongolia & provincial road \\
\hline
\end{tabular}


factors. These factors include the state of the driver and the passengers, door open time, window open time, etc. According to previous works, the factors can be categorized into human factors and objective factors $[2,3]$. Each of these factors is closely related to the escape method and survival rate.

Objective factors, such as the complexity of emergency exit handling, play an important role in emergency fire traffic accidents and draw the attention of researchers to the coach evacuation factor analysis. According to the ignition place, scholars and safety organizations aim at the Emergency Exits (EE) evaluation for evacuation efficiency improvement, including the number of EEs, the location and distribution arranged in the vehicle $[4,5]$. The behavior of occupants in the accident is thought of as the other important factor for coach evacuation analysis. Due to the knowledge, the driver always plays a role as an instructor and danger inspector in the evacuation process similarly to the aviation survival process [6-9]. When facing a dangerous situation, evacuation is more challenging due to different age, gender, and psychological endurance ability and survival skills of different passengers. This effect is especially more pronounced in the long queue circumstances $[10,11]$.

For the purpose of behavior classification and estimation, a part of methods can be used in behavior research. All of these methods can be divided into two categories: the parametric model method and the non-parametric model method [12]. The disadvantage of the parameter model-based method, such as regression analysis, is that the predefined underlying relationships between different variables could produce an erroneous estimation of the accident likelihood. The classification and regression tree (CART) is a powerful tool used for factor classification and ranking by importance [13]. It has been widely used in the crash mechanism and accident frequency analysis, traffic flow estimation and injury severity estimation [14-16]. Clear rules and simple calculation make this method more efficient for discrete values. As for the continuous target attributes, the variable should be discretized at the first step. In behavior research, CART also plays an important role when it come to the physical activity research, and the training data set can be acquired by various groups of questionnaire surveys and subject experiments. Yannakouliz adopted this method to examine the physical activity of children and obtain the relations between different factors affecting children's quality of diet [17]. Skedgell and Kearney applied CART to study the absenteeism behavior in relation to different demographic and academic risks [18]. The aforementioned studies shared the same merit in that the number of attributes was not too large, which helped to avoid unreliable estimations with too many attributes taken into consideration. Therefore, this paper extracted only three variables that can be used to describe the head rotation to decide whether this action was an effective solution for exit searching.

As mentioned earlier, estimating bus evacuation results is a huge challenge because the escape probability is affected by a combination of tremendous factors. To consider all these factors, the Bayesian network is proposed to calculate the escape probability based on prior, conditional and posterior probabilities $[19,20]$. In order to analyze the whole evacuation network and combine it with a dynamic process, the ordinary Bayesian network is developed into a Dynamic Bayesian Network (DBN). Compared with the DBN, the traditional Bayesian network is limited when handling the time-variant operation environment such as changing temperature and changing rate. This DBN method is widely used in risk and probability estimation. Yonas proposed this method to assess the risk elements connected with the drilling waste management by updating the potential risks [21]. In the evacuation research field, Sarshar and Jaziar apply this method to analyze the evacuation time during a ship fire, including the trend of the rescue process and evacuation time [22]. Furthermore, a spatio-temporal probability model integrating crowd and hazard dynamics was built according to different fire points and route selections on the ship. In these DBN research papers, an important common ground exists that the values used in the conditional probability table can be obtained either from official data or expert suggestions. This probability acquisition method solves the problem of insufficient data in DBN prediction to some extent, which is one of the most important problems in data analysis. The dynamic evacuation details, like those applied to ship evacuation, still need to be developed. To address this problem and estimate the result in an emergency situation, a DBN based risk assessment method for individual coach evacuation and factors of importance affecting the escape procedure were analyzed in the last part. 
The paper is organized as follows: Section 2 adopted the analytic hierarchy process (AHP) to build an objective system of escape requirements and rank the different factors in the evacuation process. Then the CART was adopted to classify and estimate the efficiency of exit searching through head-turning behavior. Section 3 built a DBN for the coach escape process to express the behavior of passengers and escape dynamics. In section 4, using the experiment method, the behavior of passengers was collected over time. Then, a DBN was built for coach evacuation to present the behavior of passengers and escape dynamics. Section 5 concluded the estimation results of the coach escape process using this DBN method.

\section{AHP-BASED PRIORITIZATION OF REQUIREMENTS}

Due to the high-density capacity in shuttle carriages, which is a special phenomenon in cities with high population density, the injury and death rate will be much higher in emergency accidents. Hence, the integration of vehicle condition with passenger behavior, which could improve vehicle safety, is very important.

When it comes to factors important for coach evacuation, more than 30 types of factors were investigated and organized in a hierarchical structure. It is of great difficulty to produce an accurate prediction with so many correlated factors despite a comprehensive presentation for this complicated process. To address this problem and concentrate on the most important features, the preliminary task was to define variables and then to identify the importance of these features. Table 2 shows the specific parameters in this analytic hierarchy process and concludes the meaning of these parameters.

To carry out the AHP method, an expert questionnaire is used to judge the importance of each of the two different factors. Matrix $A_{1 k}=\left[a_{i j}\right]_{n_{i} \times n_{i}}(k=1,2, \ldots, 5)$ denotes the pairwise comparison matrix with 14 alternative factors, whereas matrix $A_{2}=\left[b_{i j}\right]_{5 \times 5}$ refers to the 6 criteria factors. The element $a_{i j}$ and $b_{i j}$ are specified by domain experts and the range of values uses a scale from zero to nine according to different degrees of importance. The positive number $n_{i}(i=1,2)$ denotes the dimensions of matrix $A_{1 k}$ and $A_{2}$.

To verify all the comparison results, a consistency test is carried out for this correlation matrix. Allowing for this consistency test, a normalized matrix $\mathbf{w}$ in which all the columns are eigenvectors is produced according to Equation 1. $\lambda_{\max }$ is the maximum eigenvalue of the matrices $A_{1 k}$ and $A_{2}$. Then, the consistency ratio C.R. $=$ C.I./R.I., consistency index C.I. defined in Equation 2, and $R . I=1.98\left(n_{i}-2 / n_{i}\right)$, are employed to judge the consistency. If C.R. is no more than 0.1 or the index C.I. is equal to zero, the level of consistency is acceptable. Once the consistency check is proved to be infeasible, the expert rating content should be optimized and adjusted again to find the appropriate consistency matrix. In the end, the factors ranked in the last few places are considered to be negligible in order to simplify the hierarchy. The ultimate weights of the 14 alternatives are included in Figure 1 as follows. For simplicity, the alternative of the driver guidance efficiency (DGE) is taken as an example to show the calculative process. The pairwise comparison matrix of the driver disposal skill (DDS) is

Table 2 - Notation for different variables organized in a hierarchical structure

\begin{tabular}{|c|c|c|c|c|c|}
\hline Criteria & Alternatives & Description & Criteria & Alternatives & Description \\
\hline \multirow{3}{*}{ FIS } & IB & inflammable belongings & OEE & AW & aisle width \\
\hline & FEE & fire extinguishing equipment & \multirow[b]{2}{*}{$\mathrm{EBE}$} & EHA & emergency hammer \\
\hline & ATM & anti-fire materials & & CEDW & $\begin{array}{l}\text { convenience to open the emergency } \\
\text { door and top window }\end{array}$ \\
\hline \multirow{4}{*}{ OEE } & DOT & door open time & \multirow{2}{*}{ DDS } & DER & driver emergency guidance level \\
\hline & $\mathrm{RDA}$ & reasonability of door arrangement & & DGE & driver guidance efficiency \\
\hline & WOT & window open time & \multirow{2}{*}{ PDS } & PER & passengers emergency guidance level \\
\hline & SDW & step depth and width & & ESE & exit searching efficiency \\
\hline
\end{tabular}

Note: FIS - fire ignition and spread speed; OEE - ordinary escape method in an emergency; EBE - escape by breaking method; DDS - driver disposal skill; PDS - passenger emergency skill; 


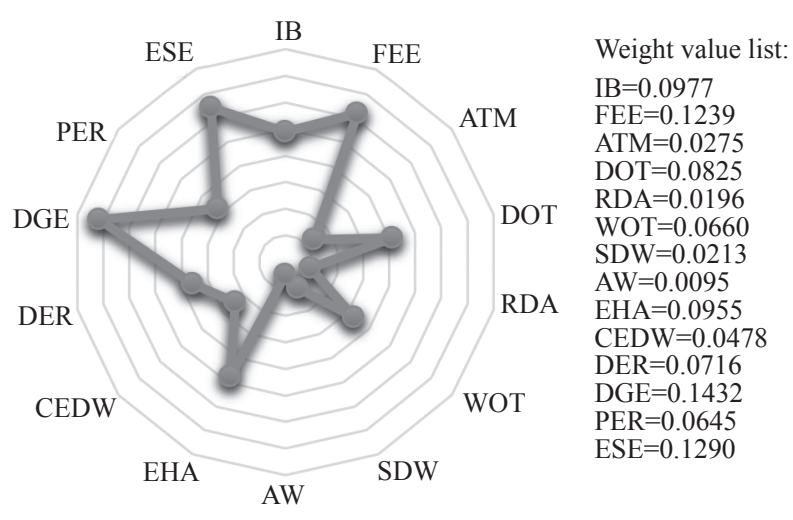

Figure 1 - The weight of factors in a mesh figure

assigned as $A_{14}=\left[\begin{array}{cc}1 & 1 / 2 \\ 2 & 1\end{array}\right]$. The maximum eigenvalue can be obtained as $\lambda_{\max }=2$ and the corresponding normalized eigenvector is $w=\left[\begin{array}{ll}0.3333 & 0.6667\end{array}\right]^{T}$. It can be seen that the weight of the DGE is 0.6667 . Multiplied by the weight obtained from the criteria, the final weight coefficient can be seen in Figure 1.

$A w=\lambda w$

C.I $=\frac{\lambda_{\max }-n_{i}}{n_{i}-1}$

It is obvious that the driver guidance efficiency (DGE) and the exit searching efficiency (ESE) have the largest effect on the whole evacuation system due to their important role in the escape decisions. On the other hand, the aisle width, step depth, step width, and reasonable door arrangement constitute the least important factors. This is mainly due to the narrow range of design size of these parameters. In order to learn the dynamic behavior of the participants during the escape in emergency conditions, a novel motion capture and behavior analytical method were introduced, which laid a foundation for the following probabilistic analysis.

\section{CART-BASED ESCAPE EFFICIENCY ANALYSIS}

Classification and regression tree is considered to be an efficient method in data mining fields. It is a feature of binary trees where the parent node is integrated with only two children nodes. Less computation burden, continuous and discrete attribute processing capacity and factor importance evaluation account for the superiority of this tree-based method. In the escape process research, capturing the exit searching by passengers and drivers is a tough issue especially for the prediction model construction. To deal with this difficulty, in this paper the attribute variables that can describe the exit searching efficiency were selected, and the CART model to predict the efficiency was built.

To build the CART in the passenger escape condition analysis, the following two steps elaborate on the process of model building.

\section{Step 1 -CART escape efficiency estimation}

CART is a convenient method for binary attribute classification problems. The tree grows from the root node to the leaf node according to a split criterion which can reduce the resubstituting estimate of the tree misclassification cost. In the CART model, the Gini heterogeneity index is proposed and defined as follows to show impurity of a node

$i(t)=1-\sum p^{2}(i \mid t)$

where $i(t)$ is a measure of impurity, $p(i \mid t)$ denotes the proportion of class $i$ in node $t$. To obtain the best tree model, all the possible splitter thresholds have to be tried and ultimately make the most impurity decrease such as

$\max \Delta i\left(t, t_{l}, t_{r}\right)=\max \left(i(t)-p_{l} i\left(t_{l}\right)-p_{r} i\left(t_{r}\right)\right)$

where $p_{l} i\left(t_{l}\right)$ and $p_{r} i\left(t_{r}\right)$ stand for the chance of instance falling into the left and right child nodes multiplied by the Gini index of the left and right child separately. Using this recursive process in each node and splitting rules, the ultimate CART model can be obtained with the maximum negative gradient direction of impurity.

\section{Step 2 - CART Optimal Pruning}

The pruning method used in the CART model is based on the optimal pruning which is featured by the losing function

$$
C_{\alpha}(T)=C(T)+\alpha|T|
$$

where $C_{\alpha}(T)$ is the total loss with parameter $\alpha$ of subtree $T, C(T)$ is the estimation error according to a given training set, $\alpha$ denotes the compromise between model complexity and model fitting, $T$ denotes any subtree. The parameter $g(t)$ is a computed variable w.r.t a node $t$ and subtree rooted by node $t$.

$g(t)=\frac{C(t)-C\left(T_{t}\right)}{\left|T_{t}\right|-1}$

where $T_{t}$ denotes the subtree of $t,\left|T_{t}\right|$ denotes the number of terminal nodes in $T_{t}$, and $C\left(T_{t}\right)$ denotes the loss of subtree $T_{t}$. When the round $i$ of pruning begins, the complexity parameter $\alpha_{i}$ is assigned to be infinity first. Then the cost-complexity decrease $g\left(t_{i}\right)$ or each node can be calculated and the least $g\left(t_{i}\right)$ (if 
not infinity) is assigned to the complexity parameter $\alpha_{i}(i=0,1,2,3, \ldots, n)$. Then the corresponding sub-tree is pruned as $T_{t}$ and the next pruning round is repeated as described.

After pruning, the relatively accurate and simplified CART model is able to estimate the escape efficiency. Based on the escape efficiency, the variation of the passengers' exit searching efficiency condition was extracted through the DBN theory dynamically as follows.

\section{DBN-BASED EVACUATION ASSESSMENT METHODOLOGY}

The Bayesian Network has been qualified for the complexity of uncertain system reasoning reduction and supplies a numerical presentation for probability estimation. To obtain the posterior probability, in Bayesian Network the directed acyclic graph (DAG) interconnects different attributes and classes with nodes and arcs using the Bayes theorem. In this research, we would like to find the posterior probability of escape on the condition that the vehicle, passengers and drivers are in a certain selected state.

Taking the dynamic relationship into consideration, the dynamic Bayesian network is built to evolve over time. With this method, two transition matrix - the state transition matrix and the output transition matrix - were selected according to different time orders. The first order of the Bayesian process is always taken as a Markov process, which means that the present state is only connected with the current step. In general, a DBN can be defined as $\left(B_{0}, B_{\rightarrow}\right) . B_{0}$ denotes a Bayesian network which shows the original time probability distribution $P\left(Z_{0}\right) . B_{\rightarrow}$ denotes a Bayesian network between two different intervals and shows the conditional distribution of these two time slices which can be expressed as follows:

$$
P\left(Z_{t} \mid Z_{t-1}\right)=\prod_{i=1}^{N} P\left(Z_{t}^{i} \mid \pi\left(Z_{t}^{i}\right)\right)
$$

where $Z_{t}^{i}$ denotes the node $i$ at time $t, \pi\left(Z_{t}^{i}\right)$ presents the parent node of $Z_{t}^{i}$

Considering the transient variation of the participants' condition, all variables were separated into temporal and ordinary parts. In the coach evacuation model, the driver escape state and the passenger escape state are the two most important time-variable factors which are always changing in the escape process. Hence, the state transition matrix can be expressed as follows:
$\mathbf{X}=\left[\begin{array}{llll}x_{01} & x_{02} & \ldots & x_{04} \\ x_{11} & x_{12} & \ldots & x_{14} \\ x_{21} & x_{22} & \ldots & x_{24} \\ x_{31} & x_{32} & \ldots & x_{34}\end{array}\right]^{T}$

where $x_{i j(j=1,2)}$ denotes different escape states of passengers and $x_{i j(j=3,4)}$ denotes the escape guidance of drivers in different time intervals.

As to this scene, the dynamic Bayesian network is employed to estimate the escape probability according to the CART classification results of the participants' exit searching efficiency. Integrated with multi-time-slices variable, the Bayesian theory can be expressed as follows:

$$
\begin{aligned}
& P(Y(t) \mid \boldsymbol{\delta}(t-1), \boldsymbol{\varphi})=\frac{P(\boldsymbol{\delta}(t-1), \boldsymbol{\varphi} \mid Y(t)) P(Y(t))}{P(\boldsymbol{\delta}(t-1), \boldsymbol{\varphi})} \\
& \boldsymbol{\delta}=(t-1)=\left(x_{1}(t-1), x_{2}(t-1)\right) \\
& \boldsymbol{\varphi}=\left(z_{1}, \ldots, z_{n}\right)
\end{aligned}
$$

where $\delta(t-1)$ denotes the temporal part in the DBN network, and in this paper the degree of emergency training knowledge is the constant. Vector $\varphi$ denotes the time-independent array in the DBN network which consists of variables $z_{1}, \ldots, z_{\mathrm{n}}$. Variable $x_{1}(t)$ is the driver guidance efficiency and $x_{2}(t)$ is the exit searching efficiency during coach evacuation. Variables $z_{1}, \ldots, z_{\mathrm{n}}$ stand for the vector of time-independent variables. In this paper, the time-independent variables include fire ignition and spread speed (FIS), ordinary escape methods in emergency (OEE), escape by breaking method (EBE), driver emergency guidance level and passenger emergency guidance level. The likelihood function $L(\boldsymbol{\delta}(t-1), \boldsymbol{\varphi} \mid Y(t))=P(Y(t) \mid \boldsymbol{\delta}(t-1), \boldsymbol{\varphi}) \quad$ and the priority probability $P(Y(t))$ can be estimated either by experiment or evaluation of the domain expert according to the variable design.

The unroll structure of the evacuation Dynamic Bayesian Network is shown in Figure 2. For the temporal part, the participants' exit searching

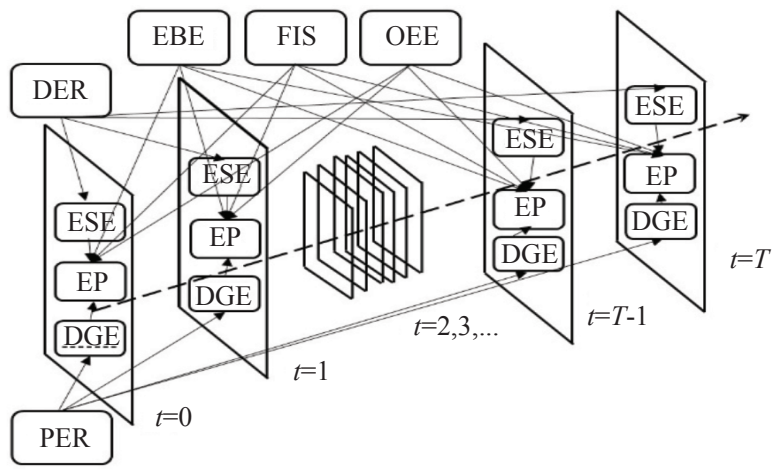

Figure 2 - The DBN with unrolled temporal nodes and time-independent nodes 


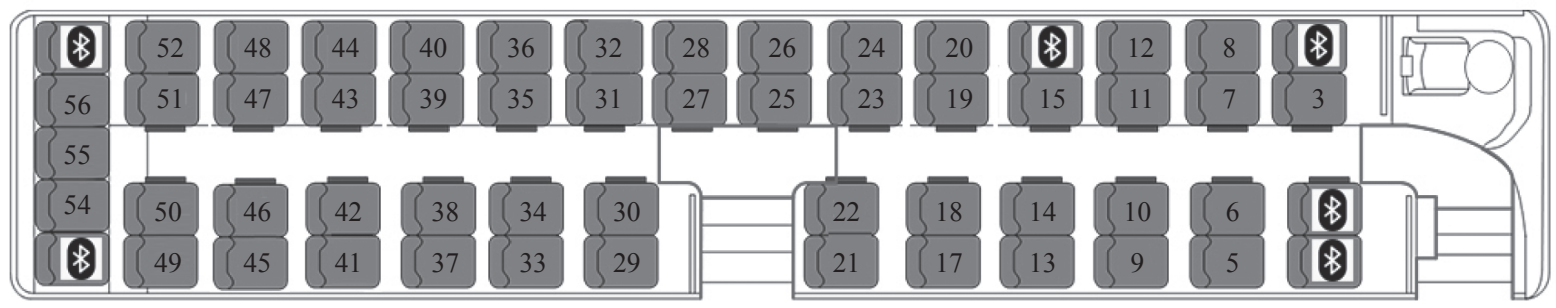

Figure 3 - The layout of seat arrangement

efficiency (ESE), driver guidance efficiency (DGE) and escape probability (EP) increased with time evolution. The other time-invariant factors, such as the driver emergency guidance level (DER), escaping by breaking method (EBE), passengers emergency guidance level (PER), ordinary escape method in emergency (OEE), and fire ignition and spread speed (FIS), have a fixed effect on the escape probability without being in relationship with the other time slices. As can be seen, the results of the experiment depend on the marginal probability of temporal and time-invariant factors. At the second time slice, the temporal node probability is added iteratively by the transition matrix and the time-independent parent nodes.

In this research, the GeNIe and temporal interfaces, which integrate the static attribute with the dynamic attributes, are introduced. GeNIe serves as an envelope tool for the Bayesian probability calculation with the clustering algorithm especially for posterior probability, which is considered to be the fastest known exact algorithm. After importing the original data set, the selected network with arcs and nodes can be learned, and then the posterior probability is supplied.

\section{EXPERIMENT STUDY}

An escape experiment in a 12-meters-long coach was carried out to simulate the accident realistically. In this coach with 57 seats, transducers were bound to the heads of the passengers in the front seat and the back seat as shown in Figure 3. The merit of this experiment setup lies in waiting for a sufficient period of time to record the escape behavior using the Bluetooth equipment. In each test, the start time and the finish time were marked by the acceleration jitter as mentioned before. The transducers on the middle seat between the front and the rear gate (most of them were not marked in Figure 3), such as No. 16, were used to monitor and record how long it takes for the passengers in the middle of the coach to form an evacuation queue under emergency circumstance for further research.

Head holding behavior was assumed to be the most important characteristic because this action reflects the searching exit behavior of the passengers and negative psychology such as restlessness and slowness. With the data derived from the LMPS system in Figure 4, the raw data of head rotation was preprocessed, and head holding duration was deduced. Concerning the collected data and the recorded video, experts were asked to evaluate the efficiency of the exit searching behavior based on their mental tools

Figure 5 shows the original data of head rotation during the escape period from getting up to getting off the coach. At first, the vertical acceleration suddenly increased following the horizontal head rotation velocity, which is shown in the stage 2 of Figure 5. With respect to the second period of stage 2 , during the long queue scene, the passengers were moving slowly through the aisle space. Under these circumstances, passenger behaviors were different according to the individual state of mind, from which we can mine the relationship between behaviors and escape efficiency. When entering the third stage, the passengers were gradually

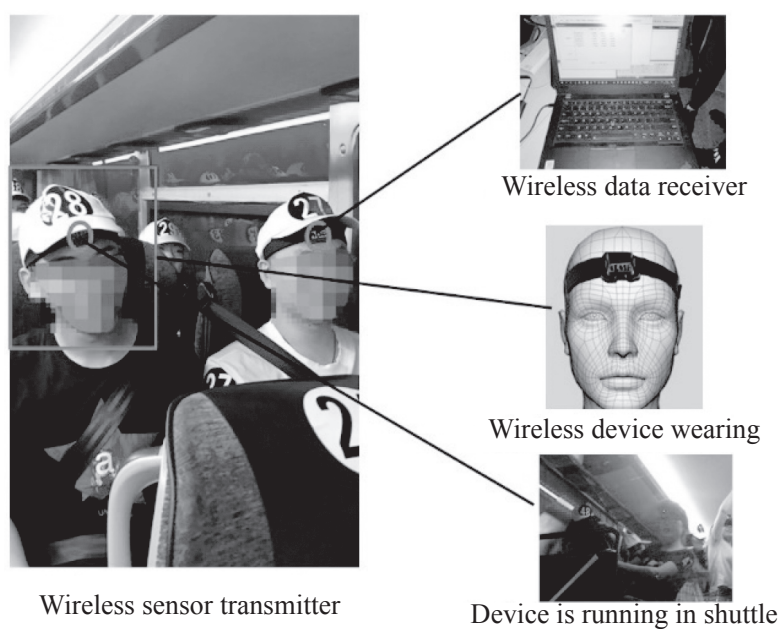

Figure 4 - Wearable Bluetooth signal equipment in the experiment 


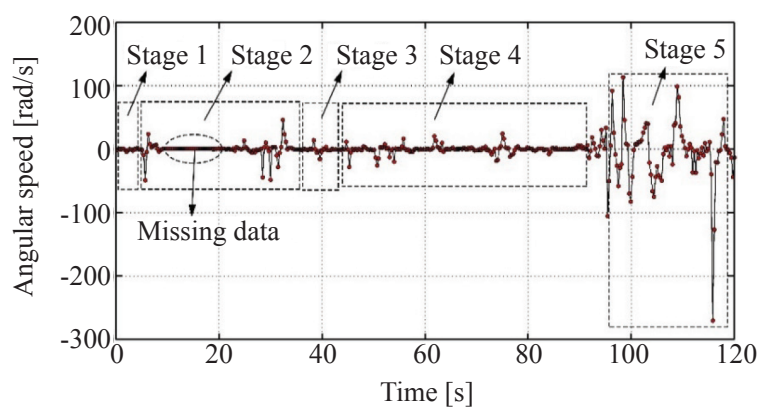

a) Head rotation speed

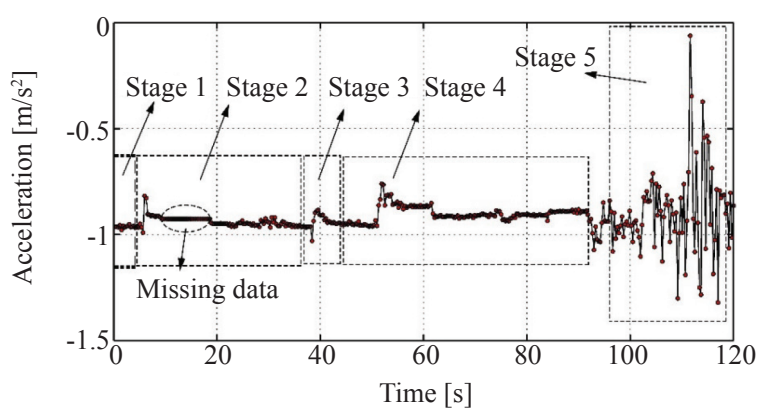

c) Head rotation vertical acceleration

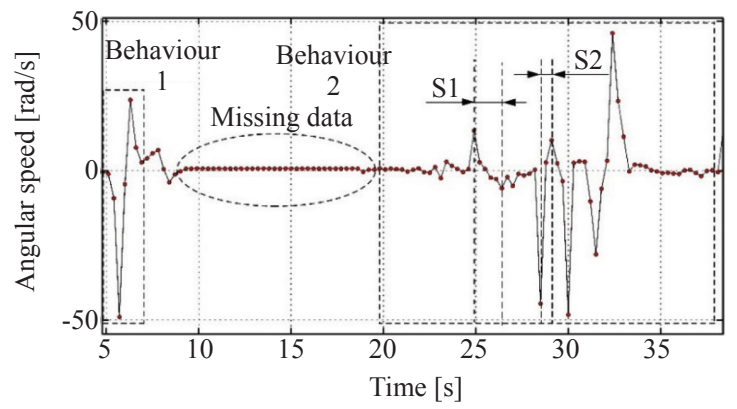

b) The enlarged view of head rotation speed in Stage 2

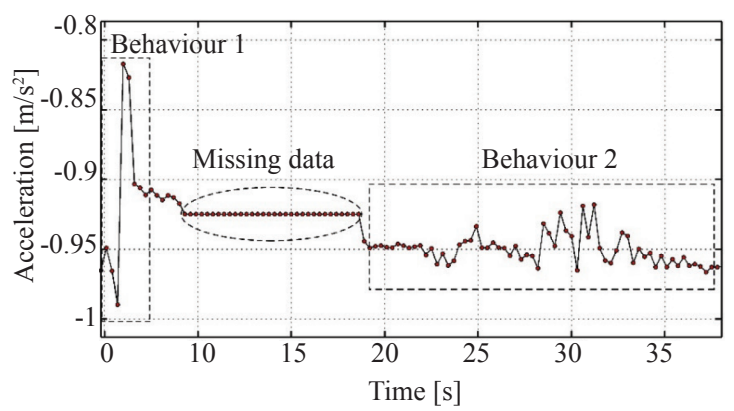

d) The enlarged view of acceleration in Stage 2

Figure 5 - Data presentation from LMPS

approaching the door and the vertical acceleration fluctuated for the debarkation. In order to study the escape behavior in the long queue circumstances, which is common in coach accidents, we focused on the second stage mentioned before, and built a classification and regression tree (CART) model to learn and estimate the efficiency produced by different escape behaviors.

As shown in Figure 5, the first stage denotes the period before the escape signal was triggered and the passengers were behaving as usual. Stage 4 and stage 5 show the state in which the passengers had escaped the coach and the equipment was removed from the participants. In Figure 5, there is a piece of data missing in stage 2 which is a common issue with Bluetooth equipment, so the data in this stage have to be deleted. In Figure 5b, Behavior 1 stands for the behavior of getting up from the seat which is a sign of escape start. To describe the preprocessing procedure, two typical characteristics are shown in Figure $5 b \mathrm{~S} 1$ and S2. S1 and S2 stand for the moment when the head began to rotate. The period between these two moments is the interval in which the head was still. This interval denotes how long the passenger searched for the exits in one direction. By collecting the duration, head rotation moment and head rotation velocity, the data were transferred to the CART model as input variables.

\subsection{CART method based participants escape state research}

The passenger escape state used in this paper refers to the ability to find different escape directions and the factors only interacted with time history, but not the coach structure. Three important variables, the head rotation moment, head rotation duration and head rotation speed were collected and preprocessed as model input variables. The passengers' exit searching efficiency, classified as efficient or inefficient, was evaluated by domain experts and set as model output. With this method, we were able to obtain much more training data because in each experiment, the available data were extracted once the three parameters constituted a valid head-turning process.

After ten-fold cross-validation, the absolute error was as small as $4.66 \%$ and the root relative squared error was $27.128 \%$. The detailed tree model is shown in Figure 6. The model shows that these three different factors interact with each other, and the escape state varies according to different head-turning moments.

To investigate the relationship between the selected three variables, a special plot matrix was employed to describe the data distribution in Figure 7. It can be seen that the combination of two variables does not present an explicit classification result. 


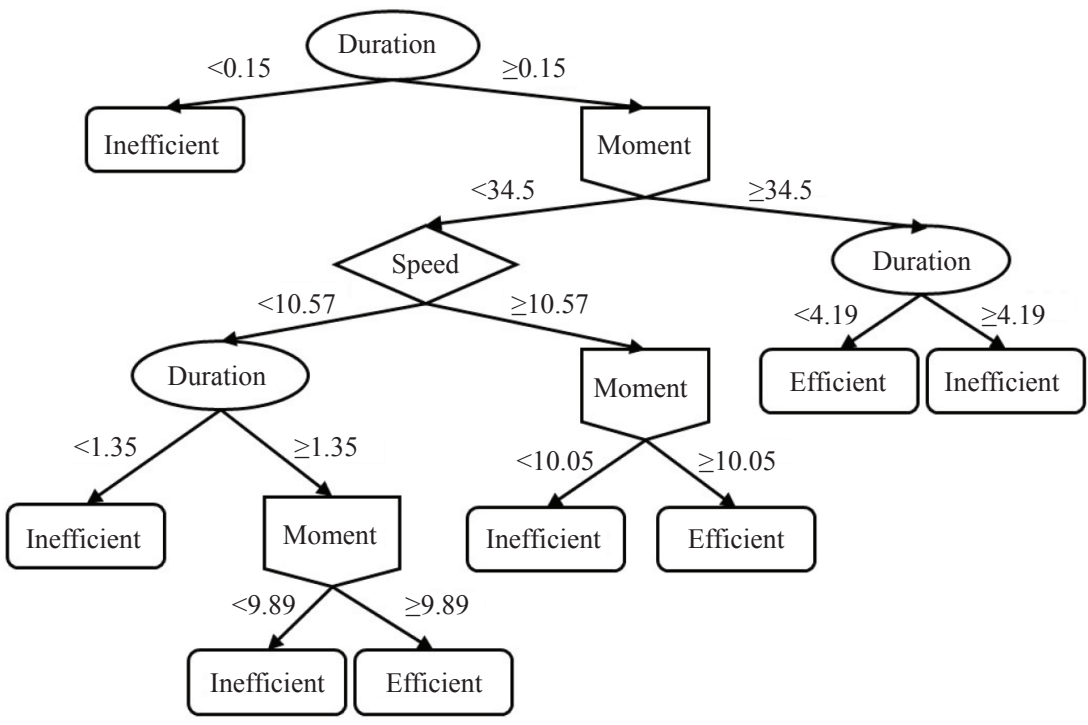

Figure 6-Exit searching efficiency CART model

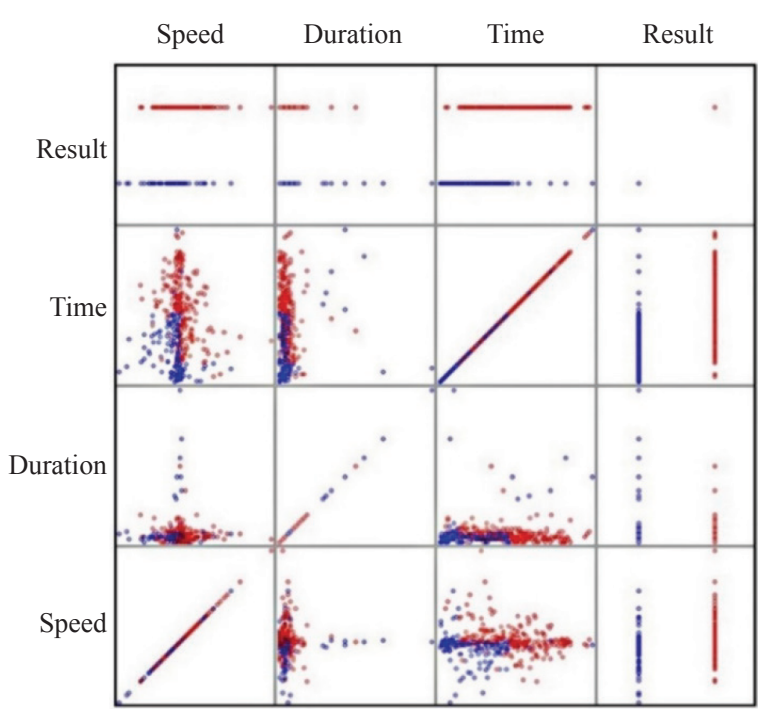

Figure 7 - Plot matrix for three variables

Eventually, the result of the three-variable model using CART is able to evaluate the exit searching efficiency and shows a more apparent classification result compared with the two-variable model. Furthermore, to demonstrate the effects of the selected three-input variables, the receiver operating characteristic (ROC) curves of different variable combinations were plotted in Figure 8. It was shown that the three-variable model owns the best true positive rate against the false positive rate. According to the area under the curve (AUC), the three-variable model AUC is 0.9695 , which is larger than the other two-variable models.

The threshold value of different classification methods is contrasted and compared in Figure 9. As shown in the figure, the Naive Bayesian and
Hoeffding trees have unfavorable classification effects due to the large false-positive rate (FPR) and small true-positive rate (TPR) along the ROC curve. However, the other tree structure methods, such as CART, Random Tree and C4.5 have a much larger AUC value than the aforementioned two methods. The summary of classification characteristics is

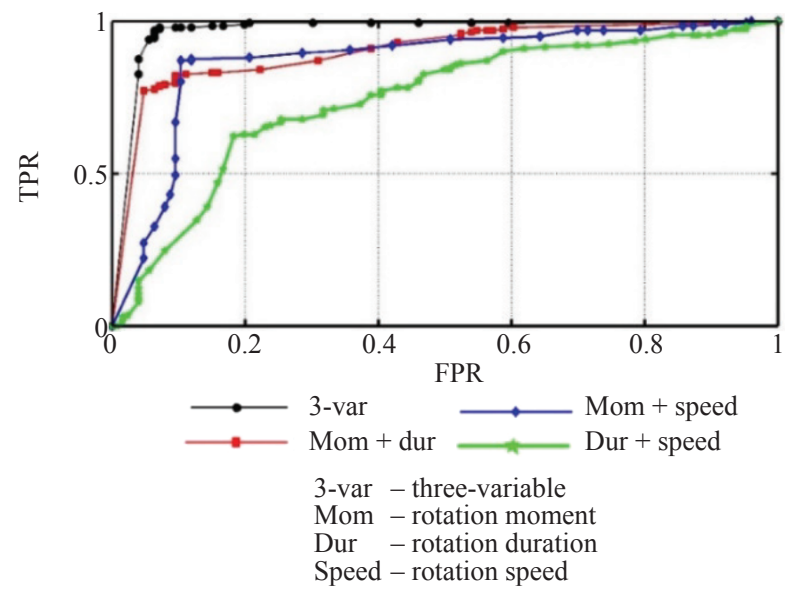

Figure 8-ROC curve of different variable combinations

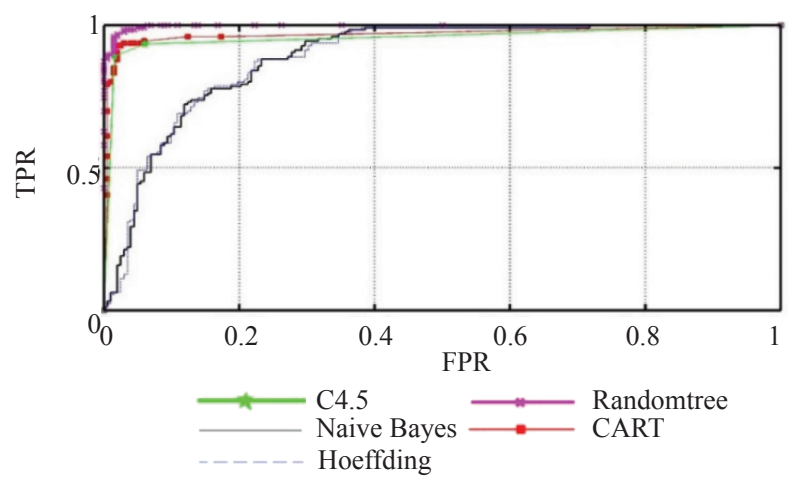

Figure 9-ROC curve of different method 
Zhou C, Zhao X, Yu Q, Huang R. Dynamic Bayesian Network-Based Escape Probability Estimation for Coach Fire Accidents

Table 3 - Summary of classification characteristics

\begin{tabular}{||l|c|c|c|c|c|c||}
\hline \multicolumn{1}{|c|}{ Method } & $\begin{array}{c}\text { Correct } \\
\text { rate }\end{array}$ & $\begin{array}{c}\text { Kappa } \\
\text { statistics }\end{array}$ & $\begin{array}{c}\text { Mean absolute } \\
\text { error }\end{array}$ & $\begin{array}{c}\text { Root mean } \\
\text { square error }\end{array}$ & TP rate (inefficient) & ROC area (inefficient) \\
\hline \hline CART & $96 \%$ & $91 \%$ & 0.05 & 0.20 & 0.94 & 0.97 \\
\hline C4.5 & $96 \%$ & $92 \%$ & 0.04 & 0.19 & 0.95 & 0.97 \\
\hline Naive Bayesian & $78 \%$ & $52 \%$ & 0.28 & 0.38 & 0.59 & 0.89 \\
\hline Hoeffding & $79 \%$ & $52 \%$ & 0.28 & 0.38 & 0.59 & 0.89 \\
\hline Random Tree & $97 \%$ & $94 \%$ & 0.06 & 0.14 & 0.96 & 0.99 \\
\hline
\end{tabular}

described in Table 3. It can be seen that the Random Tree method and CART have a similar performance, and the true positive rates are $96 \%$ and $93 \%$ respectively after a 10 -fold cross-validation. The Kappa statistics of CART is $91 \%$, which means that the selected method's performance is entirely different from random classification.

It is obvious that the escape state influenced by the passengers' exit searching efficiency is strongly correlated with time history. Furthermore, this time-varying parameter contributes largely to the evacuation results constructed as root node in the Dynamic Bayesian Network (DBN). Based on the classification predicted by the CART, the probability of turning to a different escape state or staying in the current state can be calculated by the DBN.

\subsection{DBN escape probability tendency research}

In this DBN construction, the time-independent conditional probability and driver guidance are mainly evaluated through the opinions of the domain experts due to lack of accident evacuation data or videos. As for the temporal nodes, the exit searching efficiency is assigned a value derived from the CART. The construction of the DBN is shown in Figure 10 using a bar chart.

Figure 11 depicts the estimated escape probability and exit searching efficiency considering the layout of the coach $-40 \%$ at the beginning, $40 \%$ in the middle, and $20 \%$ in the final stage. As shown in Figure 11, with the increment of time in the initial and in the final stage, the escape results tend to deteriorate. On the other hand, the middle escape stage shows an entirely different process according to the experiment results, which means that after 10 to 20 seconds, the passenger escape efficiency shows a noticeable rise. This is mainly due to the fact that after the initial period of panic and unawareness, the passengers start to consciously search for the available exits. After checking all known exits, it is explicitly shown that longer and slower lines seriously affect the passengers' exit searching efficiency in the final stage.

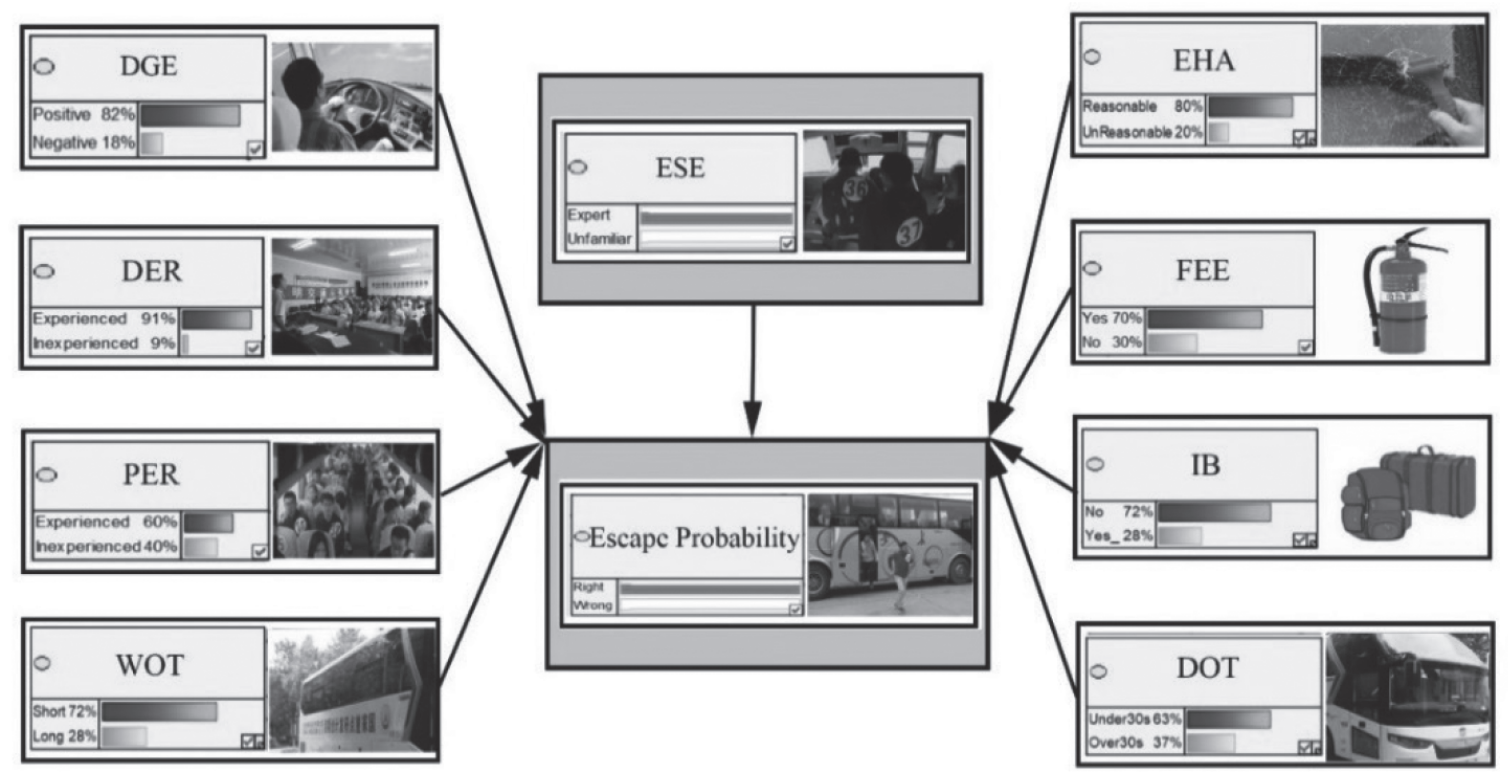

Figure 10-DBN network for the coach escape probability tendency 


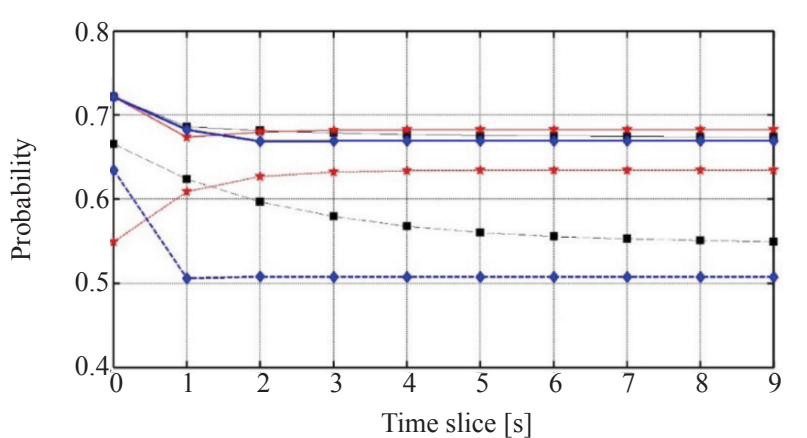

* Searching efficiency (b) _ _ Escape state (b)

$\longrightarrow$ Escape state (c) - Searching efficiency (a)

- Escape state (a)

Figure 11 - Escape probability and exit searching efficiency evolving with each time slice

In order to understand the different escape processes of different groups with various emergency training degrees, the subjects were divided into two groups. The first group received escape training before this test, and the other group was taken as a comparative group. Figure 12 reflects the exit searching efficiency and the escape probability evolving. It can be seen that the group with survival knowledge performed better and had a higher probability of effective head turning than the comparative group. From the perspective of probability, the participants with escape skills were able to maintain a steady effective exit searching probability. That is because some subjects in the comparative group performed a series of random actions and frequently followed the queue without trying to find other exits. The other reasonable explanation for this behavior is the restricted ability to find other usable exits in the case of failure to search for an exit at the current moment.

Except for passenger behavior varying with time, time-independent factors also play an important role in escape efficiency. Falling luggage, missing

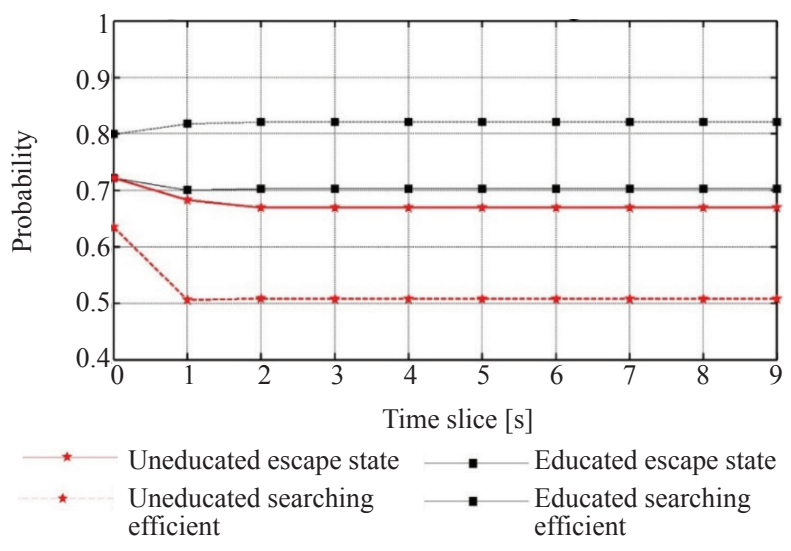

Figure 12 - Escape probability of different education background hammers and blocked doors affect the conditional probability of the escape results in coach emergency issues. To explore these three most common factors, the escape probability listed in Figure 13 was estimated under seven different factor combinations. It is indicated that the door and the emergency hammer play an important role in the escape process because this is the most common and direct measure to get an exit. Even though the falling luggage does affect the escape result, it is a controllable factor which can be handled instinctively and it has slighter effects on the results compared with the other factors. Furthermore, the low rate movement under a long queue condition reduces the risk of being tripped by luggage. As clearly shown in the figure, once the door and aisle are blocked and the hammers are out of use, the escape probability will significantly drop below 0.55 .

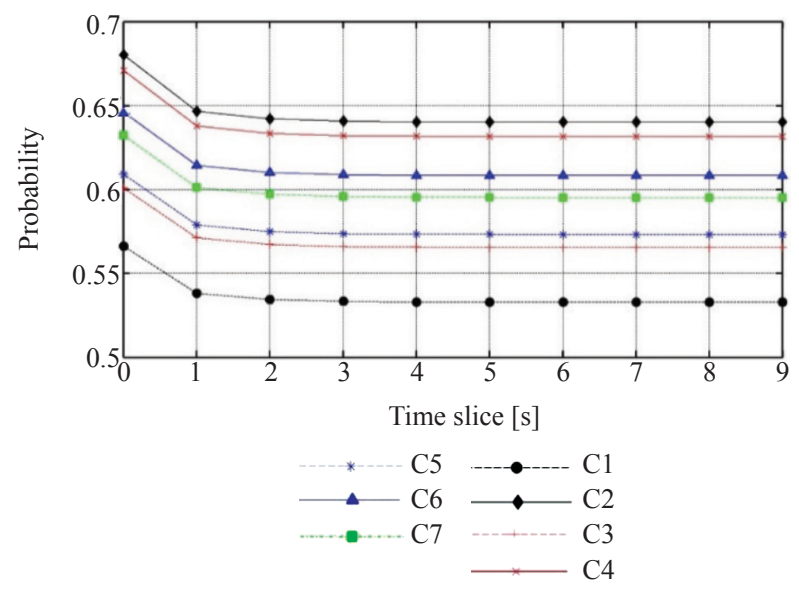

Figure 13 - Escape probability of different failure modes

\section{CONCLUSION}

This paper introduced the CART classification and DBN estimation methods to value the passenger escape state, by considering the time-evolving process of the passenger behavior. The AHP method was adopted to analyze the relevant variables that influence the escape result. Based on the factor importance ranking, nine most important factors were selected to build an escape model. In order to focus on the passenger behavior, a novel experimental method adopting wireless head transducers was employed to build the CART model. Head rotation speed, rotation moment and rotation duration were selected as input variables and the exit searching efficiency was set as the output variable. The Kappa statistic, TP rate of inefficient searching and 
AUC were 91\%, 0.94 and 0.97 respectively, and the CART classification method was verified as accurate and reasonable in this efficiency evaluation problem.

By combining temporal and time-independent variables, a DBN was built to estimate the escape results through the probabilistic method. By comparing the probability between three different stages, it can be seen that after about 10 seconds, the escape probability of the passengers shows a noticeable rise compared to the initial and final stage. Furthermore, the passengers who received evacuation training had a higher successful escape probability than the untrained passengers when it comes to low exit searching efficiency. Moreover, in typical accident circumstances, if the emergency hammers are missing and the exits are blocked, the escape probability will significantly drop below 0.55 . These inference results can be used for supplying coach inspection guidance for the transportation bureau and helping manufacturers to strengthen the vehicle escape safety.

\section{ACKNOWLEDGEMENTS}

This paper was supported by the National Key R\&D Program of China (2017YFC0803904); Special Funds for Fundamental Scientific Research of Central Universities (300102220114); Shaanxi Province Key Industry Innovation Chain (Group)-Industrial Field (2020ZDLGY16-03, 2020ZDLGY16-04). The authors would like to thank the Chang'an University Motor Judicial Authentication Center for their assistance in the accident investigation and the expert consultation process.

周辰雨, 博士 ${ }^{1}$

E-mail: feibode@hotmail.com 赵轩, 博士 ${ }^{1}$

E-mail: zhaoxuan@chd.edu.cn

余强, 博士 ${ }^{1}$

E-mail: qiangyu@chd.edu.cn

黄榕, 硕士 ${ }^{1}$

E-mail: huangrong@chd.edu.cn

1 长安大学

中华人民共和国西安市长安中路长安大学南院汽车学院

基于动态贝叶斯网络的客运车辆火灾逃生概率 估计研究

摘要

客运车辆紧急逃生研究是降低严重车辆火灾事 故伤亡率的有效手段。采用无线传感器的新型实验
方法, 通过采集头部旋转速度、头部转动时刻以及 头部转动间隔并以此作为输入构建分类与回归树模 型。基于此模型, 分类结果可以准确的对乘客逃生 出口搜寻效率进行分类。通过排除层次分析法得到 的最不重要的三个因素, 将分类与回归树得到的动 态变量及与时间无关的其它变量相结合, 构建动态 贝叶斯网络模型。仿真结果表明最高效的逃生出口 搜寻阶段是在紧急情况出现 10 秒之后, 也就是逃生 的中期阶段。在这个阶段内, 成功逃生的概率有了 明显的增加。另外, 通过接受车辆逃生教育, 成功 逃生的概率可以提升 $10 \%$ 以上。通过对比不同的逃 生失败情况, 应急锤的布置和车门的开启可靠性对 成功逃生概率的影响大于客车走道宽度的影响。基 于仿真结果, 在逃生锤丢失、车门失效以及过道受 阻的情况下, 成功逃生概率将降至 0.55 以下。

\section{关键词}

火灾逃生安全; 动态贝叶斯网络; 分类和

回归树; 逃生行为实验; 逃生概率估计.

\section{REFERENCES}

[1] Zhang DL, Xiao LY, Wang Y, Huang GZ. Study on Vehicle Fire Safety: Statistic, Investigation Methods and Experimental Analysis. Safety Science. 2019;117: 194-204. DOI: 10.1016/j.ssci.2019.03.030

[2] Zhao Z, Liang D, Yao HW. Application of Numerical Reconstruction on a Coach Fire Investigation. Applied Mechanics and Materials. 2013;444(2013): 1600-1604. DOI: 10.4028/WWW.SCIENTIFIC.NET/AMM.444-445.1600

[3] Abulhassan Y, Davis J, Sesek R, Gallagher S, Schall MC. Establishing school bus baseline emergency evacuation times for elementary school students. Safety Science. 2016;89(2016): 249-255. DOI: 10.1016/ J.SSCI.2016.06.021

[4] Lin CS, Hsu JP. Modeling and Analysis of A Bus Fire Accident for Evaluation of Fire Safety Door Using the Fire Dynamics Simulator. Cluster Computing. 2019;22(6): 14973-14981. DOI: 10.1007/S10586-018-2464-9

[5] Feng S, Li Z, Sun X. Analysis of Bus Fires Using Interpretative Structural Modeling. Journal of Public Transportation. 2016;19(3): 1-18. DOI: 10.5038/23750901.19.3.1

[6] Van Niekerk A, Govender R, Jacobs R, van As AB. Schoolbus Driver Performance Can Be Improved with Driver Training, Safety Incentivisation, and Vehicle Roadworthy Modifications. South African Medical Journal. 2017;107(7): 188-191. DOI: 10.7196/SAMJ.2017. V107I3.12363

[7] Morgul EF, Cavus O, Ozbay K, Iyigun C. Modeling of Bus Transit Driver Availability for Effective Emergency Evacuation in Disaster Relief. Transportation Research Record. 2018;2376(1): 45-55. DOI: 10.3141/2376-06

[8] Lee E-P. Analysis of the Causes of Catastrophic Damage of Goyang Bus Terminal Fire and the Preventive Measures for Similar Catastrophic Fires. Japanese Journal of Clinical Immunology. 2018;12(1): 39-48. DOI: 10.20297/JSCI.2018.12. 1.39

[9] Dadashzadeh N, Ergun M, Kesten S, Žura M. An Au- 
tomatic Calibration Procedure of Driving Behaviour Parameters in the Presence of High Bus Volume. Promet - Traffic\&Transportation. 2019;31(5): 491-502. DOI: 10.7307/PTT.V31 I5.310 0

[10] Wang J-H, Lo S-M, Sun J-H, Wang Q-S, Mu H-L. Qualitative Simulation of the Panic Spread in Large-Scale Evacuation. Simulation. 2012;88(12): 1465-1474. DOI: 10.1177/0037549712456884

[11] Larusdottir AR, Dederichs A. Evacuation Dynamics of Children - Walking Speeds, Flows Through Doors in Daycare Centers. $5^{\text {th }}$ International Conference on Pedestrian and Evacuation Dynamics, Gaithersburg, Maryland, U.S. Springer; 2011. p. 139-147. DOI: 10.1007/9781-4419-9725-8_13

[12] Chang L, Chen W. Data Mining of Tree-Based Models to Analyze Freeway Accident Frequency. Journal of Safety Research. 2005;36(4): 365-375. DOI: 10.1016/ j.jsr.2005.06.013

[13] Tavakoli Kashani A, Shariat-Mohaymany A, Ranjbari A. A Data Mining Approach to Identify Key Factors of Traffic Injury Severity. Promet - Traffic\&Transportation. 2011;23(1): 11-17. DOI: 10.7307/PTT.V23I1.144

[14] Hutabarat LT, Amren S H, Sinambela M, Limbong T. Classification of Student's Air Traffic Control Skill Using Logistic Regression. METHOMIKA: Jurnal Manajemen Informatika \& Komputerisasi Akuntansi. 2019;3(2): 166169. Available from: https://methomika.net/index.php/ jmika/article/view/80

[15] Taamneh M, Alkheder S, Taamneh S. Data-Mining Techniques for Traffic Accident Modeling and Prediction in the United Arab Emirates. Journal of Transportation Safety \& Security. 2017;9(2): 146-166. DOI: $10.1080 / 19439962.2016 .1152338$

[16] Chen C, Zhang G, Qian Z, Tarefder RA, Tian Z. Investigating Driver Injury Severity Patterns in Rollover Crashes Using Support Vector Machine Models. Acci- dent Analysis \& Prevention. 2016;90(1): 128-139. DOI: 10.1016/J.AAP.2017.07.008

[17] Yannakoulia M, Lykou A, Kastorini CM, Papasaranti ES, Petralias A, Veloudaki A, et al. Socio-Economic and Lifestyle Parameters Associated with Diet Quality of Children and Adolescents Using Classification and Regression Tree Analysis: the DIATROFI Study. Public Health Nutrition. 2016;19(2): 339-347. DOI: 10.1017/ S136898001500110X

[18] Skedgell K, Kearney CA. Predictors of School Absenteeism Severity at Multiple Levels: A Classification and Regression Tree Analysis. Children and Youth Services Review. 2018;86(1): 236-245. DOI: 10.1016/j. childyouth.2018.01.043

[19] Zwirglmaier K, Straub D, Groth KM. Capturing Cognitive Causal Paths in Human Reliability Analysis with Bayesian Network Models. Reliability Engineering \& System Safety. 2017;158(158): 117-129. DOI: 10.1016/ J.RESS.2016.10.010

[20] Sarshar P, Granmo O, Radianti J, Gonzalez JJ. A Bayesian Network Model for Evacuation Time Analysis During a Ship Fire. 2013 IEEE Symposium on Computational Intelligence in Dynamic and Uncertain Environments (CIDUE), 16-19 April 2013, Paris, French. New York, US: IEEE; 2013. p. 100-107. DOI: 10.1007/s10489-0140583-4

[21] Ayele YZ, Barabady J, Droguett LE. Dynamic Bayesian Network-Based Risk Assessment for Arctic Offshore Drilling Waste Handling Practices. Journal of Offshore Mechanics and Arctic Engineering. 2016;138(6): 5130201-5130212. DOI: 10.1115/1.4033713

[22] Radianti J, Granmo O-C, Sarshar P, Goodwin M, Dugdale J, Gonzalez JJ. A Spatio-Temporal Probabilistic Model of Hazard and Crowd Dynamics for Evacuation Planning in Disasters. Applied Intelligence. 2015;42(1): 3-23. DOI: 10.1007/s10489-014-0583-4 